Pesq. Vet. Bras. 36(2):123-130, fevereiro 2016 DOI: $10.1590 / \mathrm{S} 0100-736 \mathrm{X} 2016000200010$

\title{
Descrição anatômica de esqueletos de papagaios do gênero Amazona através da utilização de radiografias ${ }^{1}$
}

\author{
Carla C. Cavinatto², Alexandre P.R.N. Armando ${ }^{3}$, Layla K.S. Cruz², Eduardo M.M. de \\ Lima $^{2}$ e Marcelo I.S. Santana ${ }^{2 *}$
}

\begin{abstract}
Cavinatto C.C., Armando A.P.R.N., Cruz L.K.S., Lima E.M.M. \& Santana M.I.S. 2016. [Anatomical description of Amazona gender parrot's skeletons through the use of radiography.] Descrição anatômica de esqueletos de papagaios do gênero Amazona através da utilização de radiografias. Pesquisa Veterinária Brasileira 36(2):123-130. Laboratório de Anatomia Veterinária, Faculdade de Agronomia e Medicina Veterinária, Universidade de Brasília, ICC Ala Sul, Campus Universitário Darcy Ribeiro, Cx. Postal 4508, Brasília, DF 70910-970, Brazil. E-mail: misantana@unb.br

This study describes the skeleton of Amazona aestiva parrots and compared it with representatives of other Amazona species. To this end, we used 22 specimens of Amazona aestiva, two specimens each of Amazona vinacea; Amazona rhodocorythae, Amazona farinosa and one specimen each of Amazona brasiliensis and Amazona pretrei donated after natural death by Poços de Caldas Breeding Park. Full body radiographs were taken, in either the right or left lateral decubitus position, in the case of laterolateral projections, and in supine position, in the case of ventrodorsal projection. Regardless of the species, skulls of the parrots were classified as prokinetic, because of free movements in their rostral portion. In most cases, the spine consisted of: 12 cervical vertebrae, six free thoracic vertebrae, synsacrum (formed by the fusion of the last thoracic vertebrae, 7 lumbosacral vertebrae and one caudal vertebrae), five free caudal vertebrae and the pygostyle (formed by three fused caudal vertebrae). Despite punctual differences, thoracic and pelvic appendicular skeleton proved to be very similar to that observed for other genera of birds. In addition, there was no sexual dimorphism considering the anatomical characteristics of the skeletons of parrots analyzed.
\end{abstract}

INDEX TERMS: Gênero Amazona, wild birds, anatomy, psittacidae, radiographic exam.

RESUMO.- 0 esqueleto de papagaios da espécie Amazona aestiva foi descrito e comparado com representantes de outras espécies do gênero Amazona. Para tanto, foram utilizados 22 exemplares da espécie Amazona aestiva; dois das espécies Amazona vinacea; Amazona rhodocorythae, Amazona farinosa, além de um exemplar das espécies Amazona brasiliensis e Amazona pretrei, doados após morte natural pelo Criadouro Poços de Caldas. Foram realizadas radiografias de corpo inteiro, variando de decúbito lateral direito ou

\footnotetext{
${ }^{1}$ Recebido em 25 de fevereiro de 2015.

Aceito para publicação em 8 de janeiro de 2016.

${ }^{2}$ Laboratório de Anatomia Veterinária, Faculdade de Agronomia e Medicina Veterinária, Universidade de Brasília (UNB), ICC Ala Sul, Campus Universitário Darcy Ribeiro, Cx. Postal 4508, Brasília. DF 70910-900, Brasil. *Autor para correspondência: misantana@unb.br

3 Criadouro Poços de Caldas, Rua Antônio Bortalan 910, Bairro Bortolan, Poços de Caldas, MG 37704-397, Brasil.
}

esquerdo, no caso das projeções latero-laterais, e em decúbito dorsal, no caso da projeção ventro-dorsal. Independentemente da espécie, os crânios dos papagaios estudados puderam ser classificados como pró-cinéticos, por apresentarem liberdade de movimentos em sua porção rostral. Na maioria dos casos, a coluna vertebral esteve formada por 12 vértebras cervicais, seis vértebras torácicas livres, sinsacro (formado pela fusão da última vértebra torácica, 7 lombosacrais e uma caudal), cinco vértebras caudais livres e pelo pigóstilo (formado por três vértebras caudais fusionadas) e, apesar de diferenças pontuais, o esqueleto apendicular torácico e pélvico se mostrou muito semelhante ao observado para outros gêneros de aves e, inclusive, não foi possível observar dimorfismo sexual através das características anatômicas dos esqueletos dos papagaios trabalhados.

TERMOS DE INDEXAÇÃO: Gênero Amazona, aves silvestres, anatomia, psitacídeos, exame radiográfico. 


\section{INTRODUÇÃO}

A medicina de aves silvestres tem se expandido nos últimos anos graças à grande quantidade de informações existentes sobre a variedade de espécies e seu comportamento, alavancada pelo sucesso dos recentes avanços no manejo destes animais (Castro et al. 2013). Inclusive, a crescente popularidade destas aves como animais de estimação, tem criado maiores exigências quanto à obtenção de informações especializadas por parte dos médicos veterinários e, dentre elas, o exame radiográfico se destaca por ser um método diagnóstico revelador, utilizado para identificar problemas em aves com sintomas como perda de peso, vômitos e regurgitação (Junghanns et al. 1998, Silva et al. 2012).

Mas, dentre as inúmeras afecções que podem acometer as aves, as que estão relacionadas com o sistema esquelético estão entre as mais frequentes observadas na clínica. Diante disso, o exame radiográfico provou ser um método de diagnóstico por imagem essencial aplicado a essas afecções, sendo importante tanto na identificação quanto no diagnóstico diferencial entre as alterações que caracterizam uma doença óssea (Arnaut 2006). Também, o exame radiográfico é considerado um procedimento não invasivo, de baixo custo e disponível em muitos estabelecimentos veterinários, o que permite sua inclusão como exame de rotina (Aguilar et al. 2006).

Neste trabalho, o esqueleto das aves foi descrito de uma forma geral e objetiva, enfatizando a família Psittacidae, já que não existe literatura anatômica específica a respeito destes animais, apesar de pertencerem a um dos maiores grupos de aves brasileiras. Assim, o propósito deste estudo foi de oferecer informações anatômicas para a interpretação de radiografias dos diferentes esqueletos de papagaios do gênero Amazona.

\section{MATERIAL E MÉTODOS}

0 presente estudo foi realizado com base em uma amostra de trinta indivíduos adultos da família Psittacidae, especificamente do gênero Amazona, sendo 14 machos e 8 fêmeas da espécie Amazona aestiva (Papagaio-verdadeiro); dois machos das espécies Amazona vinacea (Papagaio-do-peito-roxo), Amazona rhodocorytha (Papagaio-chauá) e Amazona farinosa (Papagaio-moleiro), um macho da espécie Amazona brasiliensis (Papagaio-da-cara-roxa) e uma fêmea da espécie Amazona pretrei (Papagaio-da-serra).

As aves foram doadas, após óbito motivado por causa natural, pelo Criadouro Poços de Caldas (IBAMA 2.31.94 - 00006).

Os exames radiográficos foram realizados imediatamente após o recebimento das aves, utilizando aparelho de rádio diagnóstico convencional da marca EQUIMEX, utilizando $40 \mathrm{kv}$ e um $\mathrm{mA}$ mantido a 200. As aves foram posicionadas diretamente sobre o chassi metálico, onde foram realizadas radiografias de corpo inteiro, variando de decúbito lateral direito ou esquerdo, no caso das projeções latero-laterais, e em decúbito dorsal, no caso da projeção ventro-dorsal. Os filmes radiográficos foram revelados e fixados em processadora automática, após identificação luminosa apropriada.

Seis papagaios da espécie Amazona aestiva e um exemplar das outras espécies estudadas ainda foram dissecados, para a confirmação das imagens e das fórmulas da coluna vertebral obtidas.

A nomenclatura anatômica utilizada para a descrição do es- queleto destes papagaios está de acordo com a Nomina Anatômica Aviária (Baumel 1993).

\section{RESULTADOS E DISCUSSÃO}

De acordo com Charuta et al. (2005), em muitos trabalhos anatômicos referentes a aves silvestres, a descrição do esqueleto (ou partes dele) foi incluída de forma ocasional, portanto, genéricos quanto aos detalhes e referendando como muito semelhante ao galo doméstico, algo que nem sempre é possível, devido a grande diversidade de espécies de aves brasileiras. Por outro lado, algumas poucas referências - a maioria antigas - foram encontradas descrevendo de forma isolada os ossos de algumas regiões do corpo de aves domésticas e silvestres (Burt et al. 1930), e que foram de suma importância para a comparação com os resultados obtidos por serem anatomicamente mais precisas, justificando plenamente suas utilizações.

Neste trabalho, para a realização das radiografias, inicialmente foram feitos testes com $50 \mathrm{kv}$, como preconizado por Harcourt-Brown (2001), mas devido à extensa pneumatização encontrada nos ossos dos papagaios, esta quilovoltagem foi excessiva, o que impediu a perfeita visualização dos ossos, resultando em radiografias com estruturas muito escurecidas, devido à baixa opacidade radiográfica oferecida pela técnica utilizada. Assim, após vários testes, ficou estabelecido que as radiografias fossem obtidas com a utilização de $40 \mathrm{kv}$, pois desta forma as estruturas ósseas ficaram nítidas, ou seja, com a opacidade radiográfica adequada para a identificação detalhada de todo o esqueleto, inclusive do crânio.

No entanto, segundo Silveira (1999), a fusão dos ossos do crânio das aves durante o seu desenvolvimento dificulta o estudo individual de cada um de seus componentes e, por esta razão, para descrição e análise, as radiografias devem ser feitas priorizando regiões (Junghanns et al. 1998), as quais não foram abordadas diretamente neste estudo, sendo assim, sugerem-se a realização de estudos específicos somente para o crânio destes psitacídeos estudados, inclusive com a utilização de aves imaturas sexualmente.

Mesmo assim, apesar do crânio não ter sido extensivamente estudado neste trabalho, algumas características anatômicas específicas puderam ser observadas, como globos oculares inseridos em grandes órbitas ósseas (Machado et al. 2006), além de um maior espaço articular entre as maxilas e os ossos jugais e palatinos, o que sugere a possibilidade da realização de movimentos dorsoventrais, que vão de encontro aos descritos por Hoese \& Westneat (1996), os quais citaram serem as maxilas dotadas de mobilidade, o que facilitaria a absorção de impactos pela rinoteca (Fig.1).

Este fato é reforçado pela presença de tecido conjuntivo fibroso na articulação fronto-nasal, que pela sua pequena mobilidade (Fig.1), provavelmente faz com que as forças musculares aplicadas ao crânio sejam direcionadas principalmente ao conjunto formado pelos ossos quadrados, pterigoides, palatinos, jugais, vômer e maxilares (Gussekloo \& Zweers 1999, Gussekloo et al. 2001), ou seja, permitindo uma maior amplitude da citada movimentação dorsoventral e fazendo com que a rinoteca (porção superior da ranfoteca) desenvolva juntamente com a gnatoteca (porção 


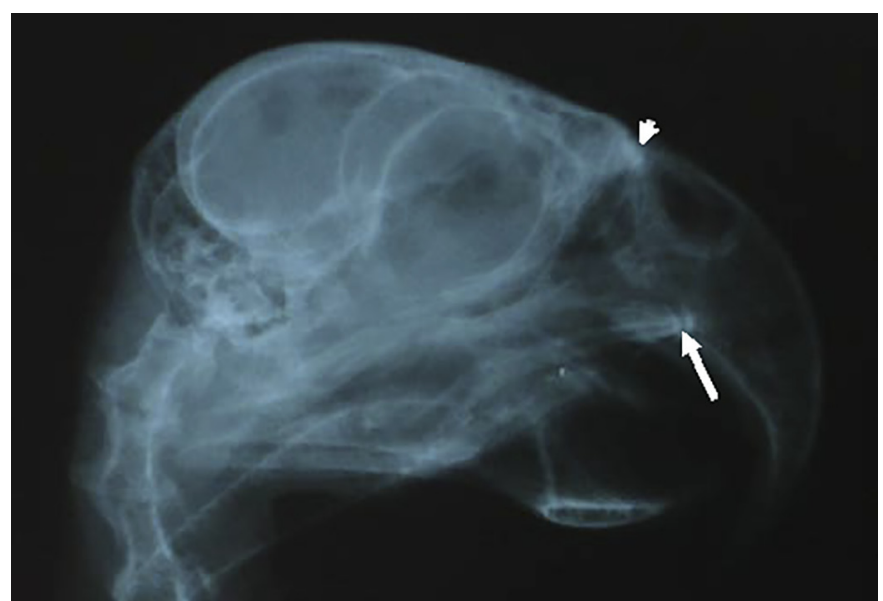

Fig.1. Radiografia do crânio de um exemplar de Amazona aestiva em projeção látero-lateral, sendo possível observar a presença de um grande espaço articular entre os ossos jugal e maxilar (seta), além da articulação contínua entre os ossos frontal e nasal (cabeça de seta).

inferior da ranfoteca) uma função semelhante à de um alicate, prendendo firmemente o alimento para que não escorregue durante sua abertura (Previatto \& Posso 2015).

Inclusive, os crânios das diferentes espécies de papagaios estudados puderam ser classificados como pró-cinéticos, já que apresentaram liberdade de movimentos na porção rostral do crânio (Zusi 2013).

Neste contexto, foi dada maior atenção às mandíbulas, pois a cinese do crânio também é dependente da movimentação do osso quadrado, que por sua vez é dependente dos movimentos de flexão e extensão da mandíbula (articulação quadrado-mandibular), pois o deslocamento rostro-dorsal do osso quadrado resulta no movimento rostral do osso pterigoide, o qual transfere o movimento para os ossos palatino, vômer e maxila, gerando assim uma elevação da rinoteca (Hoese \& Westneat 1996).

A junção deste mecanismo cinético, juntamente com a grande massa muscular associada a ele (principalmente com a mandíbula), pode explicar a importância da ranfoteca para os psitacídeos que, segundo Fecchio et al. (2008), além de ser uma estrutura especializada para a preensão de alimentos, preparo do mesmo para a deglutição, defesa e ataque, interação social e construção de ninhos, também é utilizada como elemento ativo de locomoção entre galhos de árvores ou mesmo no solo.

As análises das radiografias quanto ao número de vértebras cervicais mostraram que os papagaios da espécie Amazona aestiva, independentemente do sexo, possuem variações quanto ao seu número, já que em quinze exemplares foram observados doze vértebras (Fig.2), enquanto os outros cinco indivíduos desta espécie apresentaram onze vértebras, o mesmo número encontrado para todos os outros papagaios das espécies estudadas do gênero Amazona. É importante ressaltar que variações anatômicas são esperadas e devem ser levadas em consideração na análise radiográfica, uma vez que não representa uma anormalidade (Olson 2003).

Nas aves radiografadas, foi possível observar que o atlas é estreito e possui formato circular, estando articulado com o único côndilo do osso occipital (Krings et al. 2014). Com exceção do atlas e áxis, todas as outras vértebras cervicais apresentaram processos costais (Fig.2), maiores principalmente em C7 e C8 (Guinard et al., 2010). Também, foram encontrados processos espinhosos bem desenvolvidos de C2 a C5 e, principalmente, na última vértebra cervical (C11 ou C12), enquanto que de $\mathrm{C} 6$ a C10, este processo se pareceu mais como uma crista pouco elevada (Baumel 1993).

Ainda é válido informar que, com exceção do atlas, em todos os exemplares estudados as vértebras cervicais se mostraram heterocelosas (Fig.2), com superfícies articulares em formato de sela (Krings et al. 2014), ou seja, a superfície articular cranial do corpo vertebral se mostrou côncavo em sentido dorsoventral, enquanto que a superfície articular caudal teve curvatura oposta (Nickel et al. 1977). Tal morfologia parece favorecer a desenvoltura de movimentos como flexão lateral e extensão da região cervical nestas aves (Koerhuis et al. 2003).

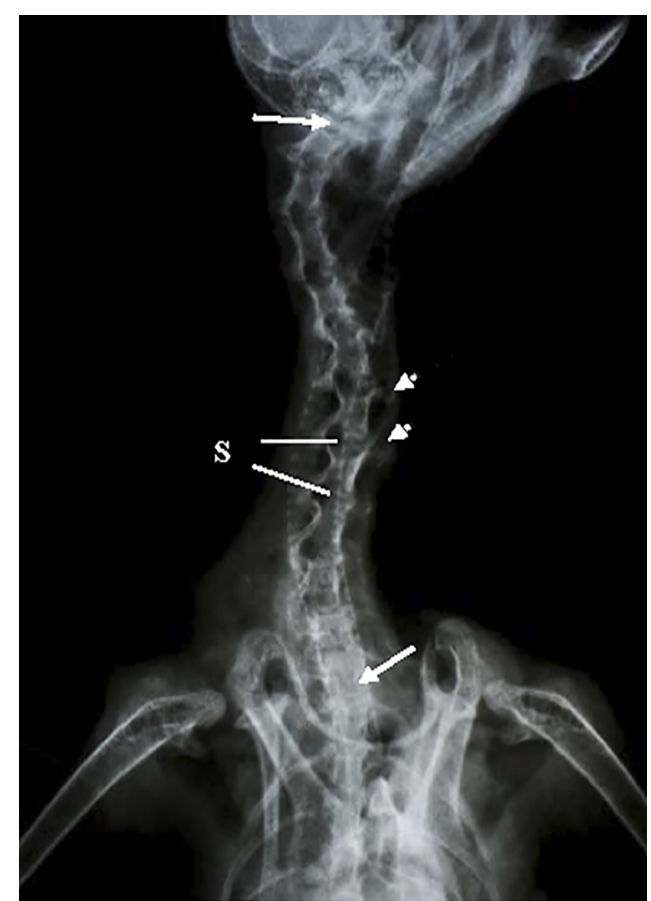

Fig.2. Radiografia da região cervical de um exemplar de Amazona aestiva, em projeção ventro-dorsal, com 12 vértebras cervicais (entre as setas cheias). Note a presença de processos costais desenvolvidos, principalmente em C7 e C8 (cabeças de seta), bem como com superfícies articulares das vértebras cervicais em formato de sela (S).

Quanto ao segmento torácico, segundo Feduccia (1986), as aves podem apresentar de quatro a seis vértebras torácicas livres, variação que não foi observada neste trabalho, já que os resultados apontaram um número fixo de seis vértebras torácicas livres (T1 a T6), portanto não fusionadas com o sinsacro (Fig.3) em qualquer das espécies de papagaios estudadas. E, apesar das vértebras torácicas também terem como característica a presença de processos espinhosos junto aos seus arcos, vale citar que estes são difíceis de serem observados na projeção ventro-dorsal, uma vez que o osso esterno e as vísceras cavitárias geram sig- 


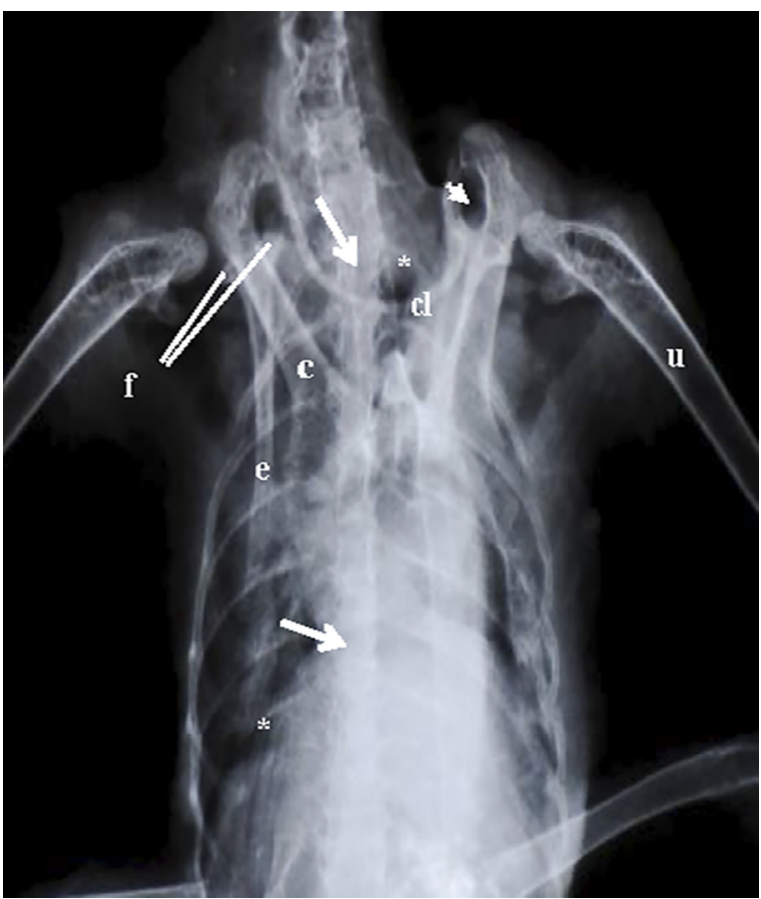

Fig.3. Radiografia da região torácica de um exemplar de Amazona aestiva, em projeção ventro-dorsal mostrando 6 vértebras torácicas livres (entre as setas), bem como seus corpos avantajados. Ainda observe a clavícula (cl), o osso coracoide (c), a escápula (e) com suas faces para articulação com os ossos citados anteriormente (f). Note a formação do canal triósseo pelos ossos relacionados (cabeça de seta), bem como a presença de sete pares costelas $\left({ }^{*}\right)$ e do osso úmero $(\mathrm{u})$.

nificante sobreposição. Inclusive, nesta projeção é possível distinguir as vértebras torácicas apenas pelos seus corpos avantajados.

Quanto à projeção látero-lateral (Fig.5), faz-se necessário informar que a visualização tanto das vértebras torácicas quanto de seus processos também foi dificultada pela

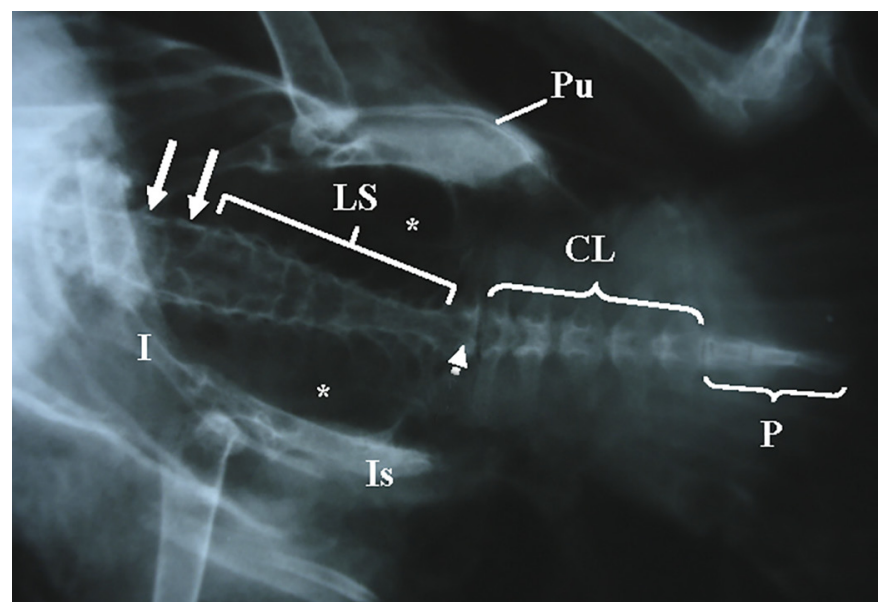

Fig.4. Radiografia da região lombossacral e caudal de um exemplar de Amazona aestiva, em projeção ventro-dorsal, mostrando T7 e T8 (setas) unidas ao conjunto de vértebras lombossacrais (LS) e primeira caudal (cabeça de seta), formando em conjunto o osso sinsacro. Observam-se também vértebras caudais livres (CL), o pigóstilo (P), os ossos ílio (I), isquio (Is) e púbis $(\mathrm{Pu})$, bem como as fossas renais $\left({ }^{*}\right)$. sobreposição das costelas, pelo seu tamanho reduzido, bem como pela grande proximidade entre elas, sendo estas, algumas modificações anatômicas que auxiliaram diminuir o peso corporal, ou seja, uma clara adaptação para o vôo (Field et al. 2013). Portanto, para confirmação do número, também foi realizada esta contagem em alguns exemplares dissecados (Fig.7), atividade que além de confirmar os resultados obtidos, também permitiu demonstrar através de comparação, que o exame radiográfico deste segmento vertebral é limitado nesta projeção.

Assim como observado para os mamíferos, nos papagaios estudados todas as vértebras cervicais (neste caso deve-se incluir atlas e axis) e torácicas articulavam-se umas com as outras por meio de junturas sinoviais, localizadas entre os processos articulares craniais e caudais de cada vértebra (Krings et al. 2014, Kumar \& Singh 2014). Mas, apesar de descritas para anatídeos (Necker 2006), não foram observadas fusões entre as vértebras torácicas em nenhuma espécie do gênero Amazona (Fig.7), fato que pode ser explicado pela presença de largos ligamentos interespinhosos, entre os processos espinhosos (Jiang et al. 1995).

Quanto à região lombosacral, foi possível observar nas diferentes espécies do gênero Amazona que o sinsacro está formado em 20 casos $(66,66 \%)$ por uma única vértebra torácica (T7), enquanto em 10 exemplares (33,33\%) de Amazona aestiva foram observadas duas vértebras fusionadas (T7 e T8); também foi notado em todos os papagaios estudados que o conjunto ósseo ainda esteve constituído por 7 vértebras lombosacrais e pela primeira vértebra caudal (Fig.4), todas da mesma forma ossificadas entre si e ao osso coxal, na área articular vertebral do ílio (parte pré-acetabular do sinsacro) e na asa do ísquio (parte pós-acetabular do sinsacro).

No entanto, observando as radiografias obtidas, ficou evidente que a individualização das vértebras formadoras deste osso deve ser criteriosa, já que o avançado processo de ossificação (sinostose) observado no sinsacro de todas as aves estudadas dificultou esta tarefa, problema que foi acentuado pela já relatada sobreposição causada pelo osso esterno e vísceras cavitárias.

Inclusive, o avançado processo de sinostose também dificultou classificar satisfatoriamente as vértebras em lombares ou sacrais na região do sinsacro, fato que definiu a utilização da nomenclatura das vértebras em lombossacrais neste trabalho (Baumel 1993). Além do mais, a variação do número de vértebras encontrada dentre as diferentes espécies de aves não chega a ser uma surpresa, já que o sinsacro é formado por uma fusão gradual de vértebras individuais durante a ontogenia (Storer 1945).

Diferentemente da região descrita anteriormente, o número de vértebras caudais livres, observadas entre o sinsacro e o pigóstilo, pode ser verificado precisamente nas radiografias um número fixo de seis vértebras caudais obtidas de 29 exemplares (96,66\%), sendo cinco livres e uma fusionada ao sinsacro (Fig.4), enquanto somente um exemplar (3,33\%) da espécie Amazona aestiva apresentou quatro vértebras livres e uma fusionada ao sinsacro.

Quando estes resultados foram comparados com outros autores (Berger 1952, Kumar \& Singh 2014, Rezk 2015), percebeu-se uma clara variação ao número encontrado, de 
4 a 6 vértebras caudais livres para estes autores e, como pressuposto por Burt (1930), pode estar relacionada com a idade e, portanto, com o número de fusões ocorridas, considerando que aves muito jovens podem apresentar um número naturalmente maior de vértebras caudais. No entanto, este fato não foi levado em conta neste trabalho, já que foram utilizados apenas papagaios adultos.

Já, quanto ao pigóstilo, da mesma forma que Hogg (1984), foram observadas somente três vértebras fusionadas para sua constituição em 26 (86,66\%) dos espécimes utilizados (Fig.4) e, em apenas $4(13,33 \%)$ papagaios da espécie Amazona aestiva, foram encontradas duas vértebras na sua formação, quadro semelhante ao descrito por Baumel (1993). Como já citado para as vértebras cervicais, estas pequenas variações encontradas são esperadas e totalmente normais dentro de um mesmo gênero (Olson 2003), já que neste caso o número de vértebras que fusionam para a formação do pigóstilo provavelmente é determinado durante a embriogênese (Berger 1952).

Assim como Tickle et al. (2007), foi encontrado para a maioria das aves sete pares de costelas, fato confirmado neste estudo (Fig.6). Porém, em dez exemplares de Amazona aestiva foi observado um par extra de costelas flutuantes, posicionado junto à asa pré-acetabular do ílio, totalizando assim oito pares, fato semelhante ao encontrado no emu (Kumar \& Singh 2014). Ainda nas costelas, os processos uncinados foram observados como processos de suas bordas caudais, orientados dorso-caudalmente, exceto no primeiro e últimos pares, além de difíceis de serem identificados devido à baixa opacidade apresentada, mesmo quando em projeção látero-lateral (Fig.5).

Quanto ao osso esterno, nas diferentes espécies de papagaios estudados, o mesmo pode ser dividido em três partes: corpo, rostro e crista (Düzler et al. 2005), dos quais as duas primeiras foram claramente identificados nas projeções dorso-ventrais e látero-laterais, enquanto a crista esternal teve sua visualização dificultada por ser muito fina e amplamente pneumatizada, apresentando portanto, menor opacidade radiográfica (Fig.5). Inclusive, as características anatômicas do esterno das aves variam de acordo com a espécie, já que depende de seus habitats e principalmente da sua capacidade de voar, portanto, aves que apresentam crista esternal desenvolvida, como os papagaios estudados, são caracterizados como bons voadores, por possuírem grande área de inserção para os músculos peitorais (Düzler et al. 2005).

Zusi (2013) afirmou que as cinturas peitorais das aves são formadas por três pares de ossos: as clavículas, os coracoides e as escápulas, que formam em conjunto, os chamados cíngulos dos membros torácicos, que unem o tronco aos membros torácicos. Estes ossos foram facilmente reconhecidos nas radiografias obtidas (Fig.3), apesar das clavículas serem extremamente delgadas em todos os papagaios analisados. Inclusive, em todos os casos, os corpos claviculares não se encontravam ossificados entre si, através de suas extremidades esternais (Baumel 1993), bem como não foi observada a presença de uma lâmina interclavicular em sua parte ventral, apesar desta característica ser muito comum para diversas famílias de aves (Höfling \& Alvarenga 2001, Zusi 2013).

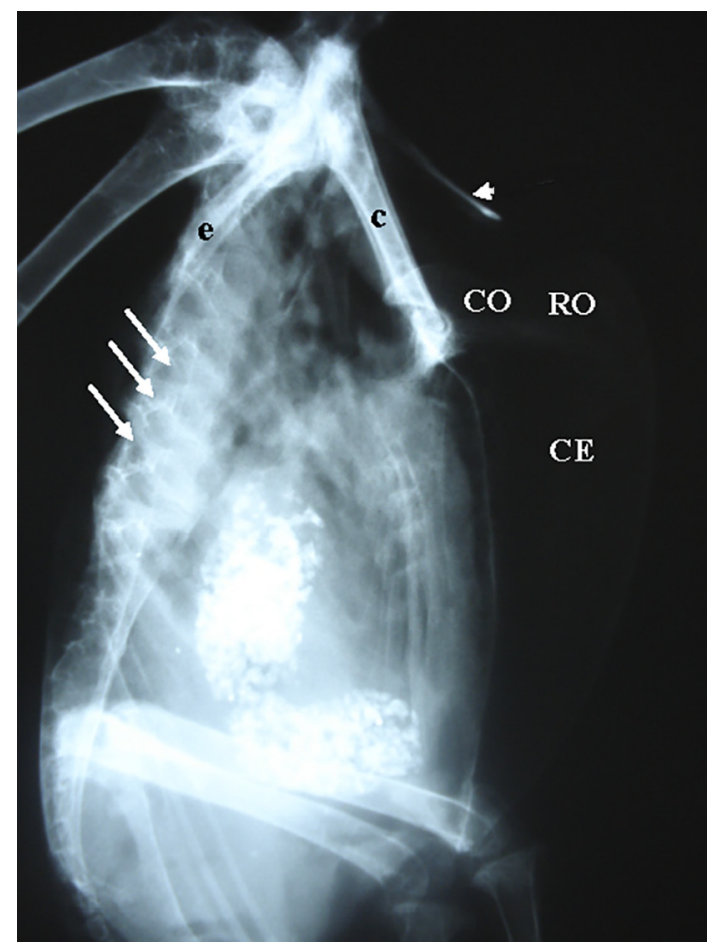

Fig.5. Radiografia em projeção látero-lateral de um exemplar de Amazona aestiva, mostrando extensa pneumatização do osso esterno (corpo [CO], rostro [RO]), principalmente na região da crista esternal (CE). Osso coracoide (C), escápula (e) e clavícula (cabeça de seta). Nota-se também uma grande sobreposição das costelas com as vértebras torácicas, dificultando sua visualização (setas).

As escápulas de todas as espécies de papagaios se caracterizaram por ter um corpo estreito, com margens dorsais e ventrais agudas, posicionados dorsalmente aos primeiros quatro pares de costelas, suas extremidades craniais se articulavam com os ossos coracoides e clavículas, formando as faces articulares clavicular, umeral e coracoidea (Baumel 1993) (Fig.3). Por sua vez, os ossos coracoides se mostraram longos e largos, portanto, representaram as bases dos cíngulos dos membros torácicos nos papagaios trabalhados (Nickel et al. 1977).

Nas espécies de papagaios estudados os processos procoracoides, situados nas margens mediais dos terços médios dos corpos dos ossos coracoides, se apresentaram bem desenvolvidos, representando os principais elementos para a formação dos canais triósseos, cujas paredes foram constituídas por escápulas e clavículas (Fig.3), para as passagens dos tendões dos músculos supracoracoideos, os quais são inseridos nas faces dorsais dos úmeros (Artoni et al. 2001, Höfling \& Alvarenga 2001), sendo responsáveis pela força impulsão da ave (Feduccia 1986).

Quanto aos ossos dos membros torácicos (asas), os úmeros se mostraram longos, com extremidades proximais largas e que se articulavam com os ossos escápulas e coracoides, seus corpos se mostraram tubulares e ocos e com extremidades proximais e distais que abrigam trabéculas ósseas (Fig.3), provavelmente para a entrada do saco aéreo clavicular, visando a pneumatização de seus interiores (Artoni et al. 2001, Zusi, 2013). 


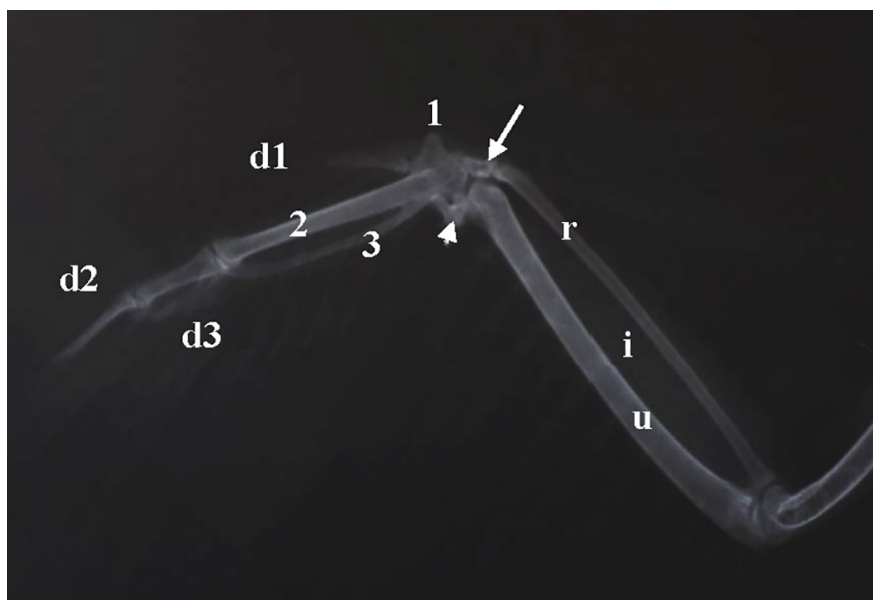

Fig.6. Radiografia do membro torácico de um exemplar de Amazona aestiva, onde se observa um grande espaço interósseo (i) entre o rádio $(\mathrm{r})$ e ulna $(\mathrm{u})$, bem como os ossos radial (seta) e ulnar (cabeça de seta) do carpo, carpometacarpos I (1), II (2), III (3) e dedos I (d1), II (d2) e III (d3).

Os antebraços dos papagaios estudados diferiram daqueles dos mamíferos por serem constituídos por largas ulnas, com olécranos pouco desenvolvidos, e por rádios muito delgados e estreitos, dispostos paralelamente e possuindo praticamente o mesmo comprimento, ficando separados por um grande espaço interósseo (Nickel et al. 1977) (Fig. 6). Os papagaios apresentaram os ossos dos antebraços um terço maiores que os úmeros, característica comum para aves voadoras, pois permite a implantação de um maior número de remiges primárias na mão e secundárias no antebraço (Charuta et al. 2005).

Como observado para outras espécies de aves, os papagaios do gênero Amazona tiveram as mãos formadas proximalmente por dois ossos cárpicos, que representam a fusão de vários outros ossos individuais (Zusi, 2013) e, de acordo com sua topografia, foram nominados de ossos radial do carpo e ulnar do carpo (Sullivan et al. 2010), sendo facilmente reconhecidos nas radiografias obtidas (Fig.6).

Distalmente aos ossos cárpicos, as mãos dos papagaios

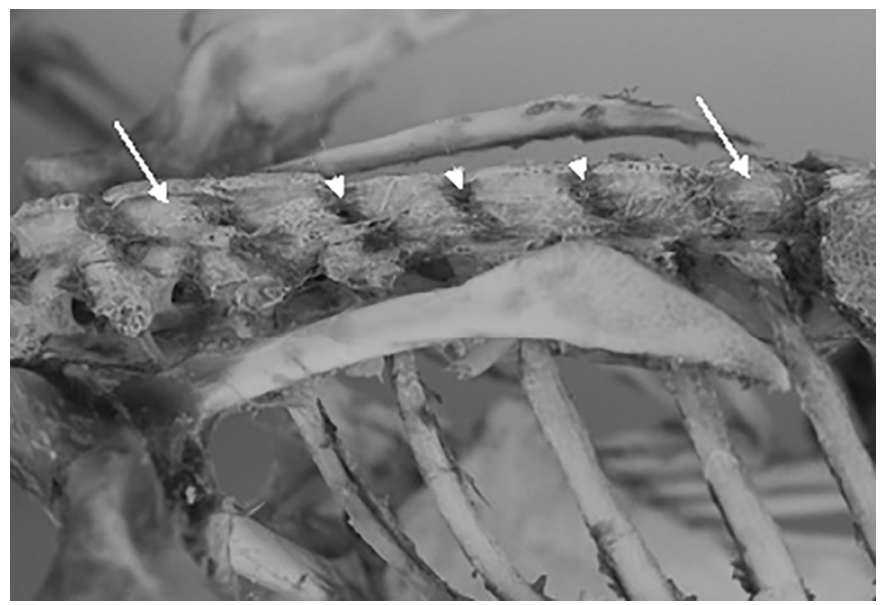

Fig.7. Vista dorso-lateral da região torácica de um exemplar de Amazona aestiva, seis vértebras torácicas livres (setas), além de largos ligamentos entre os processos espinhosos das vértebras torácicas (cabeças de seta). ainda estiveram constituídas pelos ossos carpometacarpos, resultantes das fusões das fileiras distais dos carpos com os três ossos metacárpicos. Em cada mão, os ossos carpometacarpos II se apresentaram como ossos longos, enquanto os ossos carpometacarpos I e III, por serem menores, ossificaram aos anteriores em suas extremidades proximais, formando as trócleas carpais para articulação com os ossos dos carpos (Baumel 1993, Rezk 2015). Os ossos carpometacarpos I se apresentaram como processos mediais projetados e fusionados nas extremidades proximais dos ossos carpometacarpos II, enquanto os ossos carpometacarpos III se mostraram delgados e fusionados ao II, somente nas extremidades proximais e distais, formando entre eles largos espaços intermetacárpicos (Nickel et al. 1977) (Fig.6).

A presença de trócleas carpais sugere que as mãos tenham grande flexibilidade de movimento, permitindo que sejam flexionadas quando em repouso, característica que pode ajudar a proteger as penas contra injúrias, ou da asa interferir durante deslocamentos terrestres; inclusive, este é um importante comportamento biológico que depende exclusivamente da alta flexibilidade da articulação carpometacarpica (Sullivan et al. 2010).

Correspondentemente aos três ossos carpometacarpos, foram encontrados três dedos nos papagaios estudados, onde foi observada apenas uma falange nos dedos I e III e duas falanges (proximal e distal) nos dedos II (Rezk 2015) (Fig.6). A presença de apenas uma falange no dedo I difere do típico padrão de duas falanges encontrados em pombos, galinhas e patos (Nickel et al. 1977), sendo que nos últimos, o maior tamanho deste dedo nos machos também pode ser indício de dimorfismo sexual (Charuta et al. 2005), característica que não foi encontrada nos exemplares estudados, já que esta falange em ambos os sexos de papagaios do gênero Amazona aparentaram ter o mesmo tamanho.

Quanto ao esqueleto dos membros pélvicos, ficou caracterizado que as diferentes espécies do gênero Amazona apresentaram a pelve (cíngulo do membro pélvico) formada por três ossos (ílio, ísquio e púbis) fusionados entre si e ao sinsacro (Artoni et al. 2001) (Fig.4). Por se tratarem de papagaios adultos, as superfícies ventrais das asas pré e pós-acetabulares dos ílios estavam totalmente sinistosadas aos processos transversos das vértebras pertencentes às regiões craniais e caudais do sinsacro, formando duas escavações longitudinais, as fossas renais (Baumel 1993, Rezk 2015) (Fig.4).

Em todos os papagaios radiografados os fêmures se mostraram com corpos retos, fortes, tubulares e ocos; em suas extremidades proximais foram observados trocânteres maiores pouco proeminentes e, medialmente, cabeças pequenas e hemisféricas (Rezk 2015). Suas largas extremidades distais estavam formadas por dois côndilos (laterais e mediais), separados por um largo sulco intercondilar e direcionados distocaudalmente; em suas faces craniais foram notadas duas pequenas e delgadas cristas supracondilares (laterais e mediais), onde estavam articuladas pequenas patelas ovoides e pouco visíveis nas imagens obtidas (Chadwick et al. 2014). Os côndilos laterais ainda apresentavam em sua extremidade distal uma pequena superfície articular côncava para articulação com a cabeça da fíbula, carac- 
terística esta semelhante à observada em outras espécies de aves (Baumel 1993, Rezk 2015) (Fig.8).

Os ossos tíbiotarsos (Fig.8 e 9) também apresentaram corpos fortes, ocos e com trabéculas ósseas bem definidas nas extremidades. Em suas extremidades proximais (cabeças) foram observadas duas faces articulares (laterais e mediais) ligeiramente côncavas para articulação com os fêmures. Suas extremidades distais foram marcadas pelas presenças de dois proeminentes côndilos (laterais e mediais), separados por uma profunda incisura intercondilar (Rezk 2015).

Assim como observado para outras espécies de aves (Maxwell 2008, Rezk, 2015), cada fíbula também apresentou uma cabeça bem desenvolvida, que se articulava com o côndilo lateral do fêmur, e um estreito corpo, que se articulou em todos os casos analisados com o terço proximal do corpo do tibiotarso, formando aparentemente uma sindesmose (Fig.8).

Nas espécies de papagaios estudadas, as extremidades proximais dos ossos tarsometatarsos estavam constituídas por duas cavidades articulares (laterais e mediais), separadas por uma discreta eminência intercavitária, para articulação dos côndilos dos ossos tibiotarsos (Middleton 2001, Chadwick et al. 2014). Da mesma forma como descrito para

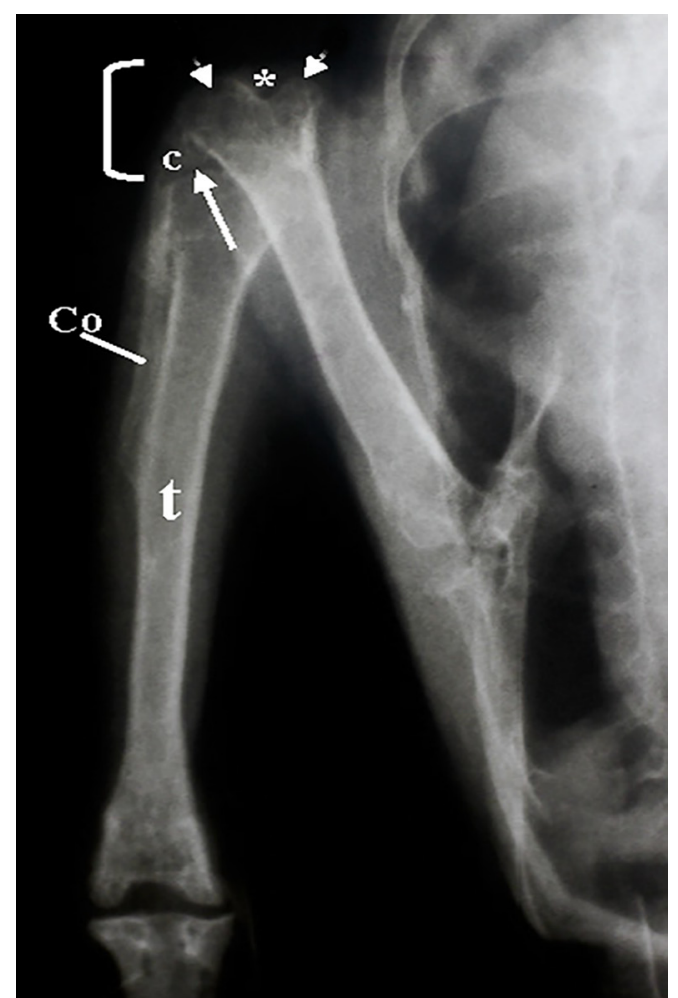

Fig.8. Radiografia em projeção dorso-ventral do membro pélvico de um exemplar de Amazona vinacea, onde se observa a articulação femurotíbiofibular (colchete), caracterizada pelas superfícies articulares proximais ligeiramente côncavas (seta) do osso tibiotarso ( $\mathrm{t}$ ), para articulação com os proeminentes côndilos do osso fêmur (cabeças de seta), separados por uma profunda incisura $\left({ }^{*}\right)$. Ainda pode-se observar a cabeça da fíbula bem desenvolvida (c), articulada com o côndilo lateral do fêmur, e um estreito corpo (Co) articulado com o terço proximal do corpo do tibiotarso, formando uma sindesmose.

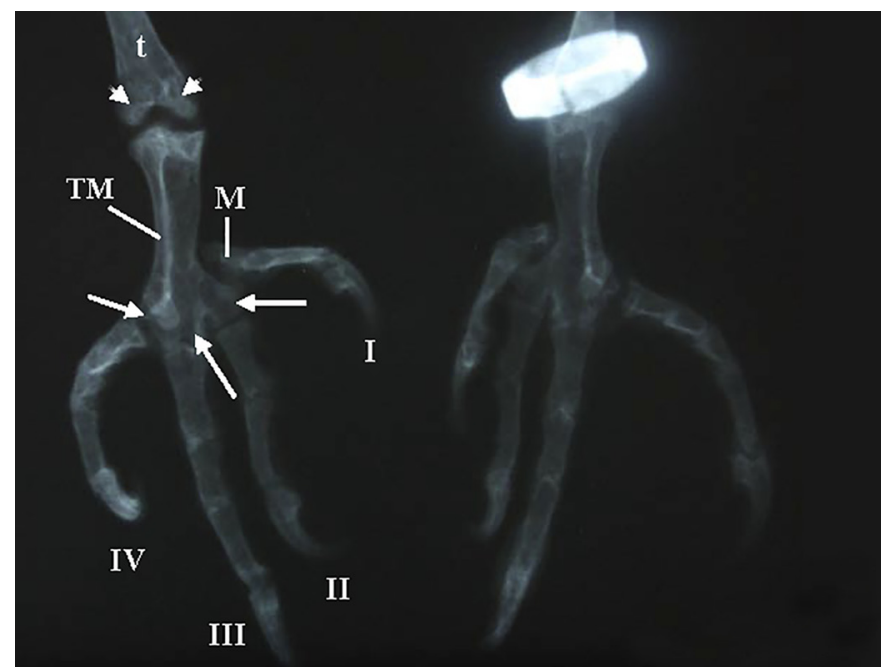

Fig.9. Radiografia em projeção crânio-caudal do osso tarsometatarso (TM) de um exemplar de Amazona aestiva, repare que suas extremidades distais apresentaram três trócleas (setas), separadas por duas incisuras intertrocleares, onde se articulam os dedos II, III e IV. O osso metatarso I (M) se articula no terço médio do referido osso, estando também articulado ao dedo I. Ainda se observa as extremidades distais do osso tibiotársico $(\mathrm{t})$, marcada pela presença de dois proeminentes côndilos (cabeças de seta), separados por uma profunda incisura intercondilar.

outras espécies de aves (Falk et al. 2011, Rezk 2015), suas extremidades distais também apresentaram três trócleas, separadas por duas incisuras intertrocleares (laterais e mediais), onde estavam articulados os dedos II, III e IV (Fig.9).

Quanto aos dedos, não foram encontradas diferenças entre as diferentes espécies de papagaios analisadas, já que todos tiveram o dedo II com três falanges, o dedo III com quatro falanges e o dedo IV com cinco falanges (Rezk 2015) (Fig.9). Também, o metatarso I, um pequeno osso individual, caracterizou-se por ser pequeno e ligado diretamente ao tarsometatarso (Falk et al. 2011), além de estar articulado com o dedo I, tendo este, duas falanges (Fig.9).

Inclusive, nos papagaios estudados, assim como em outros psitacídeos, os dedos II e III estavam alinhados paralelos e posicionados cranialmente, enquanto os dedos I e IV, também paralelos, estavam posicionados caudalmente, fatos que permite caracterizá-los como zigodáctilos (Zhang 2006). Para os psitacídeos esta configuração é essencial para que fiquem presos de forma segura aos galhos, inclusive de cabeça para baixo, além de melhorar sua estabilidade durante a busca por alimentos (Zeffer et al. 2003).

\section{CONCLUSÕES}

Para a obtenção de boa opacidade dos ossos pneumatizados, a utilização de $40 \mathrm{Kv}$ se mostrou efetiva. Os crânios dos papagaios estudados puderam ser classificados como pró-cinéticos, por apresentarem liberdade de movimentos em sua porção rostral.

Na maioria dos casos, os papagaios estudados apresentaram a coluna vertebral formada por 11 ou 12 vértebras cervicais, seis vértebras torácicas livres, sinsacro (formado pela fusão da última vértebra torácica, 7 lombosacrais e 
uma caudal), cinco vértebras caudais livres e pelo pigóstilo (formado por três vértebras caudais fusionadas).

Devido à extensa pneumatização, o osso esterno apresentou baixa densidade radiográfica. Apesar de diferenças pontuais, o esqueleto apendicular torácico e pélvico é muito semelhante ao observado para outros gêneros de aves.

Assim como para a maioria dos gêneros de aves, não foi possível observar dimorfismo sexual através das características anatômicas dos esqueletos dos papagaios trabalhados.

Agradecimentos.- Ao Criadouro Poços de Caldas, na pessoa do Sr. Moacir Carvalho Dias, por fornecer e permitir a utilização do material para a realização deste trabalho, favorecendo o aprimoramento da anatomia comparativa.

\section{REFERÊNCIAS}

Aguilar R., Hernández-Divers J. \& Hernández-Divers S.M. 2006. Atlas de Medicina, Terapêutica e Patologia de Animais Exóticos. Editora Interboock, São Caetano do Sul, p.213-229.

Arnaut L.S. 2006. Estudo radiográfico das afecções do sistema esquelético em aves. Dissertação de Mestrado em Clínica e Cirurgia Veterinária, Faculdade de Medicina Veterinária e Zootecnia, Universidade de São Paulo, São Paulo. 121p

Artoni S.M.B., Costa F.N., Pacheco M.R., Stefanini M.A., Orsi A.M. \& De Araújo M.L. 2001. Descrição anatômica do esqueleto de cisne (Cygnus melanochoriphus). Biotemas 14(2):99-108.

Baumel J.J. 1993. Handbook of Avian Anatomy: nomina anatômica avium. 2nd ed. Nuttall Ornithological Club, Cambridge. 779p.

Berger A. 1952. The comparative functional morphology of the pelvic appendage in three genera of cuculidae. Am. Midl. Nat. 47(3):513-605.

Burt W.H. 1930. Adaptive modifications in the woodpeckers. Univ. Calif. Publ. Zool. 32(8):455-524.

Castro P.F., Fantoni D.T. \& Matera J.M. 2013. Estudo retrospectivo de afecções cirúrgicas em aves. Pesq. Vet. Bras. 33(5):662-668.

Chadwick K.P., Regnault V.A., Allen V. \& Hutchinson J.R. 2014 Three-dimensional anatomy of the ostrich (Struthio camelus) knee joint. Peer]2:e706. Disponível em< https://peerj.com/articles/706.pdf> Acessado em 6 jan. 2016.

Charuta A., Bartyzel B.J., Karbowicz M. \& Kobryń H. 2005. Morphology and morphometry of the antebrachial skeleton and bones of hand of the domestic pekin duck. Vet. Med. Zootech. 29(5):26-30.

Düzler A., Özgel Ö. \& Dursun N. 2005. Morphometric analysis of the sternum in avian species. Turk. J. Vet. Anim. Sci. 30:311-314.

Falk A.R., Martin L.D. \& Hasiotis S.T. 2011. A morphologic criterion to distinguish bird tracks. J. Ornithol. 152:701-716.

Fecchio R.S., Gomes M.S., Kolososki J., Petri B.S.S., Rossi Jr J.L. \& Gioso M.A. 2008. Estudo da biomecânica oclusal e da aderência da resina acrílica auto-polimerizável (polimetilmetacrilato) em fraturas de rinoteca de tucanos (Ramphastos toco). Pesq. Vet. Bras. 28(7):335-340.

Feduccia A. 1986. Osteologia das aves, p.1680-1690 In: Getty R. (Ed.), Anatomia dos Animais Domésticos. Vol.2. Guanabara Koogan, Rio de Janeiro.

Field D.J., Lynner C., Brown C. \& Darroch S.A.F. 2013. Skeletal correlates for body mass estimation in modern and fossil flying birds. Plos One 8(11):e820DD. Disponível em< http://www.plosone.org/article/fetchObject.action?uri=info:doi/10.1371/journal.pone.0082000\&repres entation=PDF $>$ Acessado em 06 jan. 2016.

Guinard G., Marchand D., Courant F., Clerc M.C. \& Bohec C.L. 2010. Morphology, ontogenesis and mechanics of cervical vertebrae in four species of penguins (Aves: Spheniscidae). Polar Biol. 33:807-822

Gussekloo S.W.S. \& Zweers G.A. 1999. The paleognathous pterygoid-palatinum complex : a true character? Neth. J. Zool. 49:29-43.

Gussekloo S.W.S., Vosselman M.G. \& Bout R.G. 2001. Three-dimensional kinematics of skeletal elements in avian prokinetic and rhynchokinetic skulls determined by roentgen stereophotogrammetry. J. Exp. Biol. 204:1735-1744.
Harcourt-Brown N. 2001. Radiographic morphology of the pelvic limb of falconiformes and its taxonomic implications. Neth. J. Zool. 51(2):155-178.

Hoese W.J. \& Westneat M.W. 1996. Biomechanics of cranial kinesis in birds: testing linkage models in the white-throated sparrow (Zonotrichia albicollis). J. Morph. 227:305-320.

Höfling E \& Alvarenga H.M.F. 2001. Osteology of the shoulder girdle in the piciformes, passeriformes and related groups of birds. Zool. Anz. 240:196-208

Hogg D.A. 1984. The distribution of pneumatization in the skeleton of the adult domestic fowl. J. Anat. 138(4):617-629.

Jiang H., Moreau M., Raso V.J., Russell G. \& Bagnall K.A. 1995. Comparison of spinal ligaments-differences between bipeds and quadrupeds. J. Anat. 187:85-91.

Junghanns M.E.K., Kostka V.M. \& Dörsch B. 1998. Comparative studies on the diagnostic value of conventional radiography and computed tomography in evaluating the heads of psittacine and raptorial birds. J. Avian Med. Surg. 12(3):149-157.

Koerhuis C.L., Winter J.C., Van der Helm F.C. T. \& Hof A.L. 2003. Neck mobility measurement by means of the Flock of Birds electromagnetic tracking system. Clin. Biomec. 18:14-18.

Krings M., Nyakatura J.A., Fischer M.S. \& Wagner H. 2014. The cervical spine of the American Barn Owl (Tyto furcata pratincola): I. Anatomy of the vertebrae and regionalization in their S-shaped arrangement. Plos One 9(3): e91653. Disponível em <http://journals.plos.org/plosone/ article?id=10.1371/journal.pone.0091653> Acessado em 6 jan. 2016.

Kumar P. \& Singh G. 2014. Gross anatomy of axial skeleton in Emu (Dromaius novaehollandiae). Indian J. Vet. Anat. 26(2):87-91.

Machado M., Schmidt E.M.S. \& Ferreira F.M. 2006. Interspecies Variation in Orbital Bone Structure of Psitaciform Birds (with emphasis on Psittacidae). Vet. Ophthalmol. 9(3):191-194.

Maxwell E.E. 2008. Comparative embryonic development of the skeleton of the domestic turkey (Meleagris gallopavo) and other galliform birds. Zoology 111:242-257.

Middleton K.M. 2001. The morphological basis of hallucal orientation in extant birds. J. Morphol. 250:51-60.

Necker R. 2006. Specializations in the lumbosacral vertebral canal and spinal cord of birds: evidence of a function as a sense organ which is involved in the control of walking. J. Comp. Physiol. A 192:439-448.

Nickel R., Shummer A. \& Seiferle E. 1977. Anatomy of the Domestic Birds. Verlag Paul Parey, Berlin, p.3-25.

Olson S.L. 2003. Development and uses of avian skeleton collections. Bull. B.O.C. 123A:26-34.

Previatto D.M. \& Posso S.R. 2015. Cranial osteology of Cyclarhis gujanensis (aves: vireonidae). Pap. Avulsos Zool. 55(18):255-260.

Rezk H.M. 2015 Anatomical investigation on the appendicular skeleton of the cattle egret (Bubulcus ibis). J. Experim. Clin. Anat. 14 (1):5-12.

Silva J.M.M., Santos A.L.Q., Hirano L.Q.L., Pereira H.C. \& Kaminishi A.P.S. Estudo radiográfico contrastado do tempo de trânsito gastrintestinal em tucanos toco (Ramphastos toco). 2012. Revta Bras. Ciênc. Vet. 19(1):42-45.

Silveira L.F. 1999. Osteologia craniana dos Tinamidae (Aves: Tinamiformes), com implicações sistemáticas. Dissertação de Mestrado em Ciências Biológicas (Zoologia), Universidade de São Paulo, São Paulo. 109p.

Storer R.W. 1945. Structural modifications in the hind limb in the Alcidae. Ibis 87:433-456.

Sullivan C., Hone D.W.E., Xu X. \& Zhang F. 2010. The asymmetry of the carpal joint and the evolution of wing folding in maniraptoran theropod dinosaurs. Proc. R. Soc. B 3:2-7.

Tickle P.G., Ennos A.R., Lennox L.E., Perry S.F. \& Codd J.R. 2007. Functional significance of the uncinate process in birds. J. Exp. Biol. 210:3955-3961.

Zeffer A., Johansson L.C. \& Marmebro A. 2003. Functional correlation between habitat use and leg morphology in birds (Aves). Biol. J. Linn. Soc. 79:461-484.

Zhang Y. 2006. Morphology of distal tarsometatarsus and perching habits in birds. Sichuan J. Zoology 25:21-27.

Zusi R.L. 2013. Introduction to the skeleton of hummingbirds (aves: apodiformes, trochilidae) in functional and phylogenetic contexts. Ornith. Monog. 77:1-94. 